\title{
Análise de Desempenho do IEEE 802.11p: Simulações versus Experimentos Reais
}

\author{
Thales T. Almeida ${ }^{1,2}$, Lucas de C. Gomes ${ }^{1}$, Fernando M. Ortiz ${ }^{1}$, \\ José Geraldo R. Júnior ${ }^{1,2}$ e Luís Henrique M. K. Costa ${ }^{1}$ \\ ${ }^{1}$ Universidade Federal do Rio de Janeiro (UFRJ) - PEE/COPPE/GTA \\ ${ }^{2}$ Centro Federal de Educação Tecnológica de Minas Gerais (CEFET-MG)
}

\begin{abstract}
Resumo. A embarcação de dispositivos compatíveis com o padrão IEEE 802.11p no ambiente automotivo é considerado um fator crucial para alavancar a segurança e a eficiência no trânsito, o que torna fundamental a avaliação deste padrão antes de sua completa integração com sistemas reais. Entretanto, devido ao custo elevado e à pouca oferta de dispositivos comerciais, a maioria dos trabalhos envolvendo VANETs (Vehicular Ad-hoc NETworks) ainda é realizada apenas no campo das simulações. Visando comparar uma possível equivalência, este trabalho investiga os resultados de medições reais envolvendo cenários V2I (Vehicle-to-Infrastructure) e V2V (Vehicle-to-Vehicle). Para isso, foram confrontados os resultados obtidos por meio de OBUs (On-Board Units) e RSUs (Road Side Units) com os obtidos pelo simulador NS-3. Três métricas de rede foram avaliadas: o alcance máximo, a taxa de entrega de pacotes e o tempo entre recepções de pacotes. Também foi analisada a influência dos diferentes tipos de modulação permitidos no referido padrão e de diversos padrões de mobilidade. Este trabalho representa uma referência para a completa integração do padrão IEEE 802.11 p e contribui para a validação e evolução dos modelos de simulação atuais.
\end{abstract}

Abstract. The employment of IEEE 802.11p compliant devices in the automotive environment is considered a crucial factor to leverage traffic safety and efficiency, which makes it critical to evaluate this standard before it is fully integrated with real systems. However, due to the high cost and the low supply of commercial devices, most of the work involving VANETs (Vehicular Ad-hoc NETworks) is still carried out only with simulations. In order to investigate possible equivalences, this work investigates the results of real measurements involving V2I (Vehicle-to-Infrastructure) and V2V (Vehicle-to-Vehicle) scenarios. For this, results obtained using OBUs (On-Board Units) and RSUs (Road-Side Units) were compared with those obtained by the NS-3 simulator. Three metrics were evaluated: the maximum reach, the packet delivery rate and the packet inter-reception time. The influence of different types of modulation allowed in the IEEE 802.11p standard and of several mobility patterns were also analyzed. This work represents a reference for the complete integration of this standard, and contributes to the validation and evolution of the current simulation models.

\section{Introdução}

O aumento da segurança no trânsito é uma das principais motivações para a pesquisa envolvendo VANETs. De acordo com o estudo realizado pela OMS (Organização 
Mundial da Saúde) em 178 países, acidentes de trânsito são, hoje, a nona maior causa de mortes no mundo, com aproximadamente 3 mil vidas perdidas diariamente [OMS 2017]. No Brasil, conforme dados do Ministério da Saúde, somente em 2014 mais de 43 mil pessoas perderam a vida em decorrência de acidentes de trânsito [DATASUS 2017].

Além de minimizar o número de acidentes, a pesquisa envolvendo VANETs tem se tornado cada vez mais atrativa para lidar com os problemas relacionados ao crescimento desenfreado no número de veículos. Nos Estados Unidos, um relatório publicado em 2015 aponta que congestionamentos são a principal causa para um prejuízo anual de US\$160 bilhões [Schrank et al. 2015]. O tratamento dos dados gerados por OBUs (acopladas ao interior dos veículos) e RSUs (instaladas ao longo das vias), compatíveis com o padrão para comunicação veicular IEEE 802.11p, promete possibilitar a execução de ações preventivas, como identificar áreas congestionadas e disseminar alertas de segurança.

Soluções práticas têm sido propostas visando aumentar a segurança no trânsito e aperfeiçoar a gestão de congestionamentos [Amarante et al. 2015, Pinto et al. 2016]. Entretanto, antes do emprego destas tecnologias, é necessário avaliar a capacidade da rede de disseminar dados na arquitetura estabelecida. Apesar do crescente desenvolvimento de soluções implementadas em dispositivos reais, questões como o custo e a variedade limitada de dispositivos comerciais contribuem para que a maioria dos trabalhos considere apenas simulações. Poucos avaliam o desempenho do padrão IEEE 802.11p em experimentos de campo. Além disso, não se pode afirmar hoje que o comportamento obtido por uma solução simulada será equivalente ao obtido após sua implementação no mundo real.

Com o objetivo de investigar uma possível equivalência entre os resultados obtidos por simulações e experimentos reais, este trabalho propõe caracterizar o desempenho de uma aplicação de segurança no padrão IEEE 802.11p em ambos os ambientes. Baseandose na troca de BSMs (Basic Safety Messages), definidas no padrão SAE J2735, são investigados os resultados de medições reais envolvendo cenários V2I e V2V utilizando OBUs e RSUs comerciais. Os resultados destas medições são então confrontados com os resultados obtidos pela simulação do mesmo cenário no simulador de redes NS-3. Três métricas foram avaliadas: o alcance máximo obtido na comunicação, a taxa de entrega de pacotes (Packet Delivery Rate - PDR) e o tempo entre recepções de pacotes (Packet Inter-Reception time - PIR). São analisadas ainda a influência das modulações suportadas no padrão IEEE 802.11p e de diversos padrões de mobilidade empregados pelos veículos.

De acordo com nosso conhecimento, este é o primeiro estudo que procura demonstrar uma possível equivalência entre os resultados de simulações executadas no NS3 e experimentos práticos envolvendo a comunicação de dispositivos reais compatíveis com o padrão IEEE 802.11p. O restante deste artigo possui a seguinte estrutura: a Seção 2 apresenta os trabalhos relacionados. $\mathrm{Na}$ Seção 3, são apresentados os cenários dos experimentos, além dos detalhes de implementação no NS-3 e a configuração dos experimentos com dispositivos reais. A Seção 4 analisa e confronta os resultados experimentais com os de simulação. Por fim, a Seção 5 conclui o artigo e propõe os trabalhos futuros.

\section{Trabalhos Relacionados}

Diversos trabalhos na literatura têm sido desenvolvidos com o objetivo de caracterizar o desempenho de Sistemas Inteligentes de Transporte e do padrão IEEE 802.11p. Em [ElBatt et al. 2006], é realizada a análise de um sistema de prevenção de colisão dian- 
teira. Os autores desenvolveram um cenário de simulação utilizando um modelo de mobilidade realística. Todos os veículos possuem GPS de alta precisão e rádios DSRC (Dedicated Short Range Communications), e todos os pacotes possuem tamanho fixo de $100 \mathrm{~B}$. Dois cenários foram avaliados: (1) alta densidade de veículos e baixa velocidade; (2) baixa densidade de veículos e alta velocidade. Foram avaliados o tempo entre recepções de pacotes (PIR) e a taxa de entrega de pacotes (PDR). Os resultados revelam que, no cenário com alta densidade, o PIR tende a ser maior devido à maior ocorrência de perdas, sugerindo um compromisso entre PDR e PIR. Já no cenário com baixa densidade, o número de pacotes entregues é maior, desencadeando, assim, em um PIR de menor valor.

Em [Jafari et al. 2012], os autores investigam o impacto do aumento da velocidade $(80 \mathrm{~km} / \mathrm{h}$ a $130 \mathrm{~km} / \mathrm{h})$ e do tamanho do pacote $(250$ a $1.000 \mathrm{~B})$ integrando um modelo de mobilidade realística no simulador NS-2. Foram medidas a taxa de transferência de pacotes, o atraso fim-a-fim e a taxa de perda de pacotes (Packet Loss Rate - PLR). Os resultados apontam que veículos a até $138 \mathrm{~m}$ do transmissor podem receber dados. A esta distância, as diferentes velocidades não afetam os resultados. Um compromisso entre o tamanho dos pacotes com o atraso fim-a-fim e a taxa de transferência foi identificado. Outros trabalhos investigam o desempenho do padrão IEEE 802.11p por meio de simulações [Gräfling et al. 2010, Sabouni and Hafez 2012, Zhao et al. 2013]. Como mencionado na Seção 1, o custo, a variedade limitada de dispositivos e a complexidade de medições reais contribuem para que poucos trabalhos sejam desenvolvidos visando avaliar o desempenho do referido padrão em campo. Como consequência, os resultados sintéticos de simulações podem não refletir todas as situações encontradas no mundo real.

Em [Teixeira et al. 2014] foi realizada uma análise experimental do IEEE 802.11p em cenário real com dispositivos de prateleira. Os autores empregaram velocidades entre $20 \mathrm{~km} / \mathrm{h}$ e $60 \mathrm{~km} / \mathrm{h}$, além de variar o tamanho do pacote entre $150 \mathrm{~B}$ e $1.460 \mathrm{~B}$. O alcance máximo da comunicação, a taxa de transferência, a latência, o jitter, a taxa de perda de pacotes e o tempo de associação foram avaliados. Dois cenários foram implementados: (1) em laboratório, dois laptops compatíveis com o padrão IEEE 802.11p se comunicam e medem o tempo de associação; (2) em campo aberto, dois laptops em diferentes veículos avaliam as demais métricas. Os resultados apontam que o melhor desempenho foi obtido com pacotes de $500 \mathrm{~B}$, e a maior variação ocorreu nos pacotes de $1460 \mathrm{~B}$. Além disso, apesar da maior instabilidade na comunicação em movimento, os dispositivos foram capazes de transferir dados em distâncias superiores a $300 \mathrm{~m}$, com taxas excedendo $8 \mathrm{Mbps}$.

Em [Renda et al. 2016], os autores avaliam experimentalmente o PIR proveniente da troca de mensagens de informação situacional (beacons). A importância desta métrica é avaliada por meio da avaliação da probabilidade de blackouts de consciência situacional na rede veicular. Nos experimentos realizados, os autores utilizaram dispositivos compatíveis com o padrão IEEE 802.11p. Em dois cenários, foram coletados dados de dois e três veículos, em diferentes trajetos e alturas. Além das métricas PDR e PIR, também foi avaliado o número de blackouts ocorridos e a frequência com que eles ocorreram. Com base nos resultados obtidos, identificou-se que o PIR pode ser diretamente afetado pelos blackouts de consciência situacional, e que os mesmos são relativamente frequentes. Além disso, os autores concluem que o PIR não pode ser confiavelmente estimado a partir da PDR, uma vez que não foi encontrada uma correlação forte entre as duas métricas.

Em um cenário V2V, [Wang et al. 2015] definem um método de classificação de 
condições LoS (Line-of-Sight). Neste cenário, dois veículos equipados com dispositivos compatíveis com o padrão IEEE 802.11p trocam dados à taxa de $6 \mathrm{Mbps}$. São avaliados o RSSI (Received Signal Strength Indicator), PDR e latência. Já [Barcelos et al. 2014] propõem um dispositivo de baixo custo baseado no IEEE 802.11p para monitoramento de trânsito. Para demonstrar sua eficiência com base nos resultados de PLR, latência e taxa de transferência de pacotes, veículos contendo os dispositivos realizaram transmissões à taxa de $6 \mathrm{Mbps}$ e trafegaram entre $20 \mathrm{~km} / \mathrm{h}$ e $60 \mathrm{~km} / \mathrm{h}$. Por fim, [Vivek et al. 2014] conduziram experimentos com cenários V2I e V2V. Utilizando OBUs e RSUs comerciais que se comunicavam à taxa de $6 \mathrm{Mbps}$, os autores avaliaram latência, jitter e PLR.

Apesar dos trabalhos desenvolvidos com o intuito de avaliar o desempenho do IEEE 802.11p por meio de simulações e experimentos reais, nenhum estudo havia comparado os resultados de ambos os ambientes em busca de correspondências. Com foco nesta investigação, [Sassi et al. 2017] desenvolveram simulações da camada física do padrão IEEE 802.11p no software Matlab, além de medições reais utilizando o dispositivo de comunicação veicular Arada LocoMate. Nos dois ambientes, foram configurados cenários envolvendo a comunicação V2I e V2V. No cenário V2I, um dos veículos agia como uma RSU, enquanto o outro se dirigia até o primeiro em diferentes velocidades. Já no cenário $\mathrm{V} 2 \mathrm{~V}$, ambos os veículos se encontravam em movimento, percorrendo o trajeto dos experimentos em direções opostas. O impacto das diferentes velocidades dos veículos e das diferentes modulações suportadas pelo IEEE 802.11p foram avaliadas em termos de alcance máximo e PLR. Os resultados revelam que, em ambos os ambientes, as modulações associadas às taxas de dados mais baixas possuem um melhor desempenho para comunicações de longo alcance. Além disso, os resultados também revelam que a qualidade da comunicação pode ser afetada pelo aumento da velocidade dos veículos.

Como [Sassi et al. 2017], esse trabalho investiga as possíveis correspondências entre os resultados da avaliação do desempenho do IEEE 802.11p por meio de simulações e medições reais. Ao contrário da maioria das avaliações experimentais envolvendo VANETs, neste trabalho são configurados cenários reais de alta mobilidade, onde velocidades relativas de até $160 \mathrm{~km} / \mathrm{h}$ são obtidas. Além disso, a influência de diversas modulações na qualidade da comunicação é investigada. Uma importante contribuição em relação a [Sassi et al. 2017] é que a presente investigação é realizada com base nos resultados de simulações do popular simulador NS-3. De acordo com o nosso conhecimento, é a primeira vez que este tipo de estudo é realizado tendo como alvo este simulador. Ademais, além do alcance máximo da comunicação e PDR (únicas métricas avaliadas em [Sassi et al. 2017]), investiga-se também o tempo entre recepções de pacotes (PIR), buscando identificar, como [Renda et al. 2016], se a métrica possui correlação com a PDR.

\section{Experimentos para Avaliação do Padrão IEEE 802.11p}

Os experimentos consistem de simulações executadas no simulador de redes NS-3 e medições reais utilizando OBUs e RSUs Cohda Wireless (Figura 1(d)).

\subsection{Cenários dos Experimentos}

Três cenários foram definidos para as simulações e experimentos práticos.

Cenário 1 - Alcance Máximo: nesse cenário, visa-se verificar o alcance máximo da comunicação entre dispositivos IEEE 802.11p. A Figura 1(a) apresenta a configuração 
deste cenário: um veículo parado contém uma OBU em seu interior que se comunica com uma RSU instalada na extremidade da via. A via é dividida em 10 segmentos de $100 \mathrm{~m}$ e as transmissões são realizadas pela OBU com destino à RSU. Iniciando as transmissões a $100 \mathrm{~m}$ da RSU, após um determinado número de BSMs enviadas, o veículo avança de 100 em $100 \mathrm{~m}$, até se afastar $1.000 \mathrm{~m}$ da RSU (alcance máximo teórico do IEEE 802.11p).

Cenário 2 - Mobilidade Moderada: esse cenário visa verificar o efeito da mobilidade moderada no desempenho do IEEE 802.11p. Verifica-se o impacto de diferentes velocidades empregadas pelo veículo em uma comunicação V2I. O veículo trafega a $20 \mathrm{~km} / \mathrm{h}$, $50 \mathrm{~km} / \mathrm{h}$ e $80 \mathrm{~km} / \mathrm{h}$. A configuração deste cenário é apresentada na Figura 1(b): um veículo (contendo uma OBU) inicia sua trajetória a $1.000 \mathrm{~m}$ de distância da RSU e transmite continuamente BSMs a esta unidade. A análise considera as transmissões realizadas durante todo o percurso entre o veículo e a RSU, sem discriminar os resultados por segmento.

Cenário 3 - Mobilidade Intensa: por fim, esse cenário verifica o efeito da mobilidade intensa no desempenho. São utilizados dois veículos, sendo um deles o transmissor e outro o receptor, caracterizando uma comunicação V2V. A Figura 1(c) apresenta a configuração deste cenário: os dois veículos partem de cada extremidade da via, ao mesmo tempo e com velocidades idênticas, o que permite alcançar velocidades relativas maiores $(40 \mathrm{~km} / \mathrm{h}$, $80 \mathrm{~km} / \mathrm{h}$ e $160 \mathrm{~km} / \mathrm{h}$ ). Assim como no Cenário 2, a transmissão de BSMs é contínua.

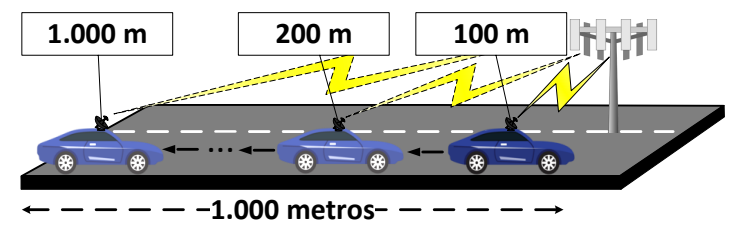

(a) Alcance máximo de comunicação.

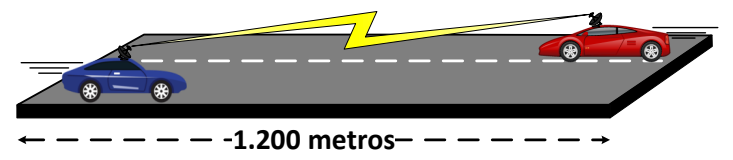

(c) Efeito da mobilidade intensa na comunicação.

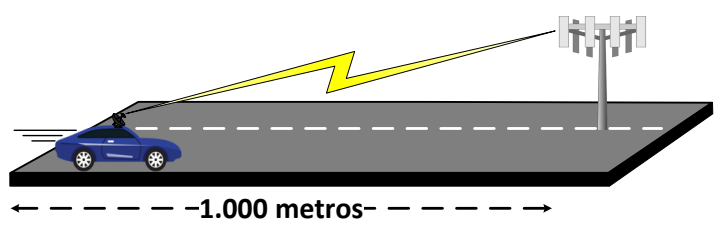

(b) Efeito da mobilidade moderada na comunicação.
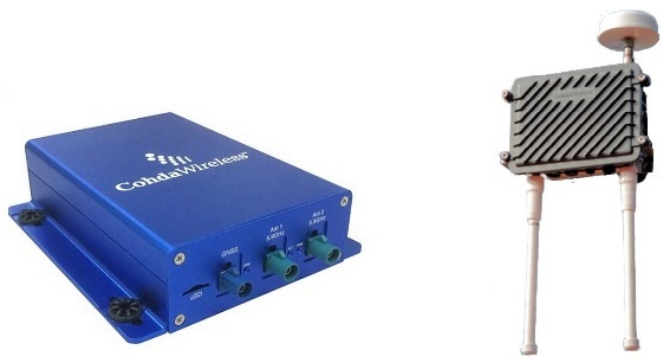

(d) Dispositivos IEEE 802.11p Cohda Wireless

Figura 1. Cenários dos experimentos e dispositivos utilizados.

A análise do desempenho do padrão IEEE 802.11p tem como base a investigação dos resultados envolvendo a PDR e o PIR. A maior parte dos estudos considera investigar apenas a PDR [Renda et al. 2016]. Entretanto, uma vez que o objetivo é caracterizar o desempenho do padrão e verificar sua eficiência especialmente em aplicações de segurança, a análise das duas métricas é justificável por demonstrar a variação da qualidade de transmissão (PDR) e, principalmente, a ausência de comunicação por longos períodos (PIR).

Nos três cenários, um estudo comparativo também é realizado para as diferentes modulações definidas no IEEE 802.11p. São oito as modulações suportadas: BPSK 1/2, BPSK $3 / 4$, QPSK $1 / 2$, QPSK 3/4, 16 QAM 1/2, 16 QAM 3/4, 64 QAM $2 / 3$ e 64 QAM 3/4, associadas, respectivamente, às taxas de 3, 4.5, 6, 9, 12, 18, 24 e 27 Mbps. Neste trabalho, 
decidiu-se por testes apenas com as taxas de 6, 12, 18 e 24 Mbps. São duas as justificativas para tal escolha: (1) a complexidade envolvendo a realização de medições reais em um cenário com grande número de permutações (nos experimentos, os veículos realizaram um percurso de aproximadamente $480 \mathrm{~km}$ ); e (2) a expectativa de que, entre taxas próximas (como 3, 4.5 e $6 \mathrm{Mbps}$ ) os resultados das métricas avaliadas sejam semelhantes. A Tabela 1 resume as características dos cenários avaliados.

Tabela 1. Cenários dos Experimentos.

\begin{tabular}{|c|c|c|c|c|c|}
\hline Cenário & Verificação Realizada & Transmissor & Receptor & Mobilidade & Taxa de Dados \\
\hline 1 (V2I) & Alcance Máximo & OBU & RSU & Estático $(0 \mathrm{~km} / \mathrm{h})$ & $6,12,18$ e $24 \mathrm{Mbps}$ \\
\hline $2(\mathrm{~V} 2 \mathrm{I})$ & Efeito da Mobilidade Moderada & OBU & RSU & 20,50 e $80 \mathrm{~km} / \mathrm{h}$ & $6,12,18 \mathrm{e} 24 \mathrm{Mbps}$ \\
\hline $3(\mathrm{~V} 2 \mathrm{~V})$ & Efeito da Mobilidade Intensa & OBU & OBU & $40,100 \mathrm{e} 160 \mathrm{~km} / \mathrm{h}$ & $6,12,18 \mathrm{e} 24 \mathrm{Mbps}$ \\
\hline
\end{tabular}

\subsection{Detalhes de Configuração das Simulações no NS-3}

Esta seção descreve o projeto das simulações realizadas no NS-3. Dado que as versões mais atuais do simulador (NS-3.27 e versões imediatamente anteriores) não possuem nenhum modelo que simule os impactos provocados pelo efeito Doppler, e dada a presença de cenários cujo objetivo é verificar a influência da mobilidade na comunicação, foi necessário utilizar e adaptar o módulo PhySimWiFi, uma derivação do NS-3 baseada no padrão IEEE 802.11. Incorporando modelos de canais sofisticados, este módulo considera os efeitos multi-caminhos de forma mais precisa, além do efeito Doppler.

Originalmente implementado para funcionar com o padrão IEEE 802.11a, foi necessário ajustar a configuração do módulo PhySimWifi de forma a torná-lo compatível com os parâmetros de camada física do IEEE 802.11p. Nesta implementação, as BSMs são transmitidas a cada $100 \mathrm{~ms}$. O canal de controle $\mathrm{CCH}$ é utilizado para transmissão de dados entre os nós que participam da comunicação. A largura de banda foi definida em $10 \mathrm{MHz}$ e o espectro de frequência na faixa de $5.9 \mathrm{GHz}$, de acordo com o padrão DSRC.

Com base nas especificações das OBUs e RSUs usadas nos experimentos, foi definida uma EIRP (Effective Isotropic Radiated Power) de $23 \mathrm{dBm}$, com ganho de antena de $6 \mathrm{dBi}$ (caso o receptor seja uma RSU) ou $5 \mathrm{dBi}$ (caso de uma OBU). O limiar de energia do sinal recebido foi configurado em $-99 \mathrm{dBm}$. Como mencionado anteriormente, a camada de enlace utiliza quatro esquemas de modulação: QPSK 1/2, 16 QAM 1/2, 16 QAM 3/4, 64 QAM 2/3, associadas, respectivamente, às taxas de dados de 6, 12, 18 e $24 \mathrm{Mbps}$.

Para simular a atenuação do sinal de rádio proporcionalmente ao aumento da distância entre o transmissor e o receptor, configurou-se o modelo de perda de propagação LogDistance. Neste modelo, o expoente de atenuação foi definido empiricamente, com base nos resultados do alcance máximo da comunicação para a taxa de $6 \mathrm{Mbps}$. Integrado ao LogDistance, foi configurado também o modelo RicianPropagationLoss, responsável por emular o desvanecimento rápido e garantir o impacto do efeito Doppler.

Para gerar a mobilidade realística dos veículos, utilizou-se o IDM (Intelligent Driver Model) [Arbabi and Weigle 2010]. As características da via simulada foram baseadas nas características físicas do cenário real. A duração definida para as simulações foi de 2.000 segundos, enquanto que cada pacote possuía $1.500 \mathrm{~B}$ de tamanho.

\subsection{Detalhes dos Experimentos Práticos com Dispositivos Reais}

As medições reais foram realizadas utilizando OBUs e RSUs modelo MK5, conforme mostra a Figura 1(d). Fabricados pela Cohda Wireless, os dispositivos possuem 
rádio compatível com o IEEE 802.11 , potência de transmissão de $24 \mathrm{dBm}$, GPS com precisão de $1.5 \mathrm{~m}$, antenas com sensibilidade de $-100 \mathrm{dBm}$, frequência de $5.9 \mathrm{GHz}$ e taxas que vão de 3 a 27 Mbps. Os dois dispositivos embarcam o sistema operacional Linux.

A comunicação consiste na transmissão de BSMs, que inclui as coordenadas geográficas do nó (obtidas por meio do GPS com taxa de atualização de $200 \mathrm{~ms}$ ), sua velocidade atual, além do timestamp da informação gerada e outras informações do veículo. Nas medições reais, as BSMs são transmitidas através do canal de controle 178, a cada $50 \mathrm{~ms}$. Assim como nas simulações, cada BSM possui um tamanho fixo de $1.500 \mathrm{~B}$.

Para a coleta das métricas, o nó transmissor armazenou o timestamp dos momentos em que as BSMs foram enviadas e suas coordenadas geográficas. O nó receptor armazenou os eventos de atualização das coordenadas geográficas e de recepção de BSMs, os tempos em que eles ocorreram e o conteúdo das BSMs recebidas. Os valores obtidos para a PDR e o PIR passaram por um processo de remoção de outliers: quaisquer valores cujas diferenças em relação à média foram maiores que dois desvios-padrões foram removidos.

As medições foram realizadas em um aeroporto desativado do município de Leopoldina - MG. Composto por uma pista de $1.2 \mathrm{~km}$ de extensão, o local demonstrou ser o ideal para o experimento: não recebe interferência de redes celulares ou WiFi e, por estar localizado em uma área predominantemente aberta, não possui presença de árvores ou edifícios que prejudicariam as condições de LoS. A Figura 2(a) apresenta a visão aérea do local, enquanto as Figuras 2(b), 2(c), 2(d), 2(e) e 2(f) apresentam os elementos envolvidos nos experimentos e fotos dos três cenários de avaliação.

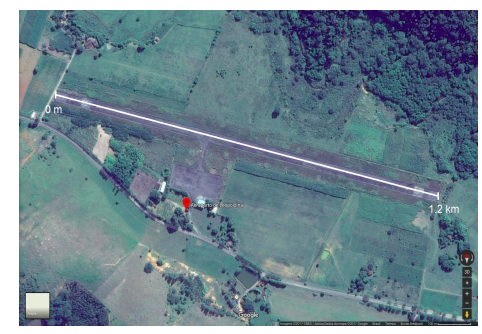

(a) Cenário dos experimentos.

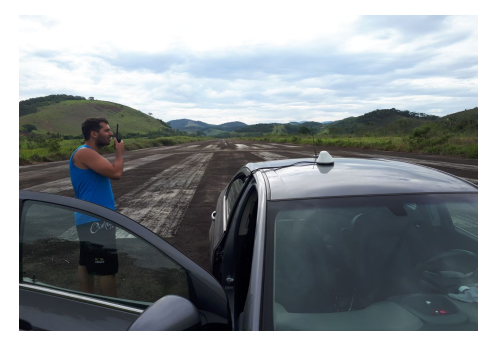

(d) Avaliando o alcance máximo.

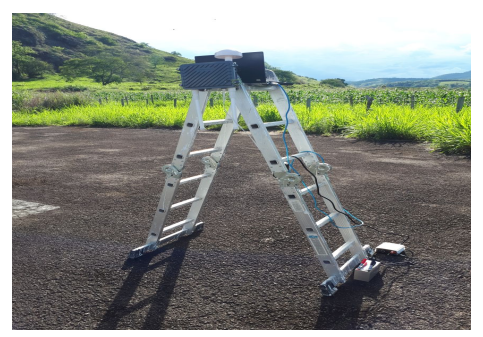

(b) RSU fixada no limite da pista.

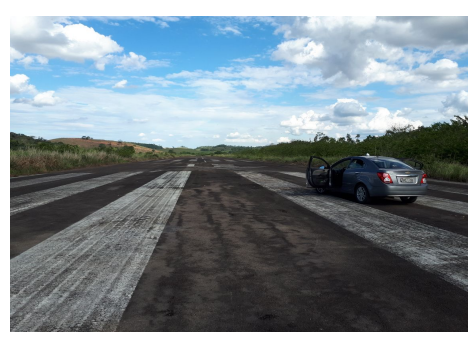

(e) Avaliando o cenário V2I.

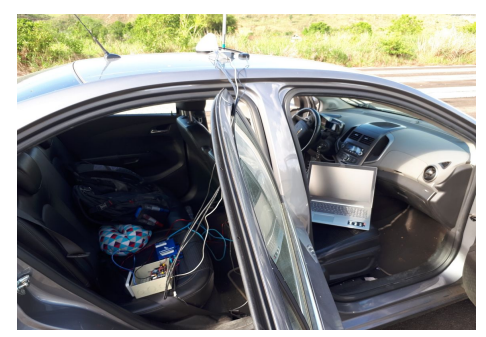

(c) OBU acoplada ao veículo.

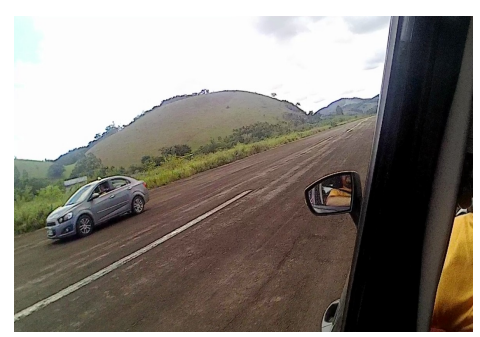

(f) Avaliando o cenário V2V.

Figura 2. Cenário dos Experimentos Práticos.

\section{Resultados}

Essa seção compara os resultados das simulações e medições reais. Em ambos os ambientes, realizou-se 10 rodadas de cada permutação dos cenários definidos na Seção 3. 


\subsection{Verificação do Alcance Máximo da Comunicação}

Este conjunto de experimentos investiga o alcance máximo da comunicação suportado por dispositivos compatíveis com o padrão IEEE 802.11p. As Figuras 3(a) e 3(b) apresentam a PDR em cada segmento de $100 \mathrm{~m}$ nas simulações e medições reais, enquanto as Figuras 3(c) e 3(d) apresentam o PIR obtido nas mesmas condições.

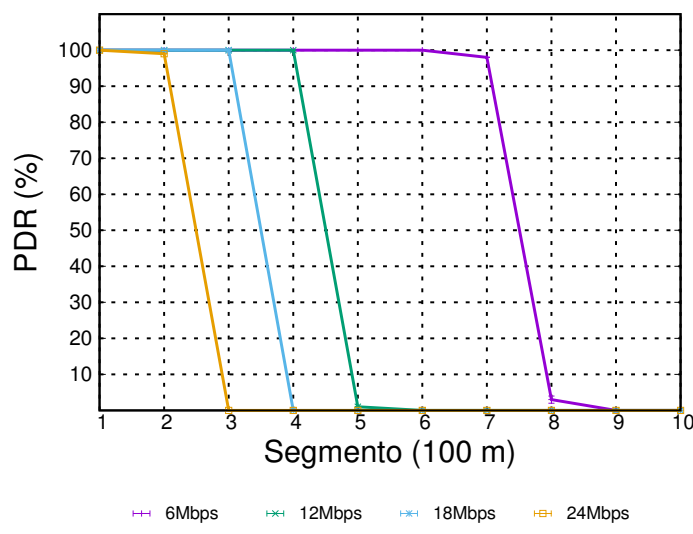

(a) PDR obtido das simulações.

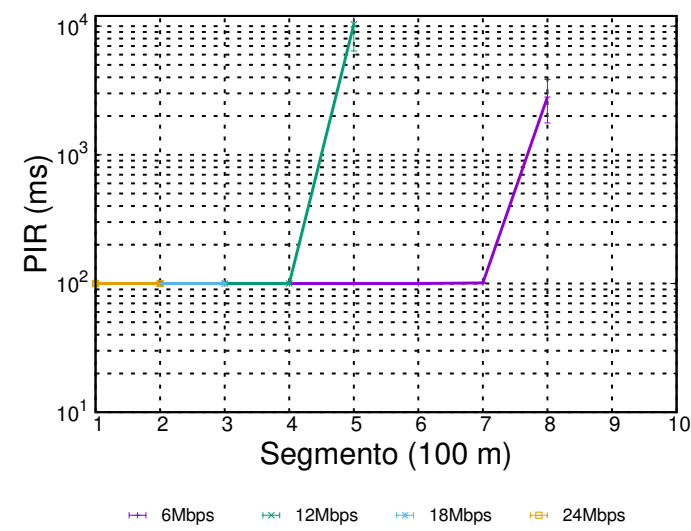

(c) PIR obtido das simulações.

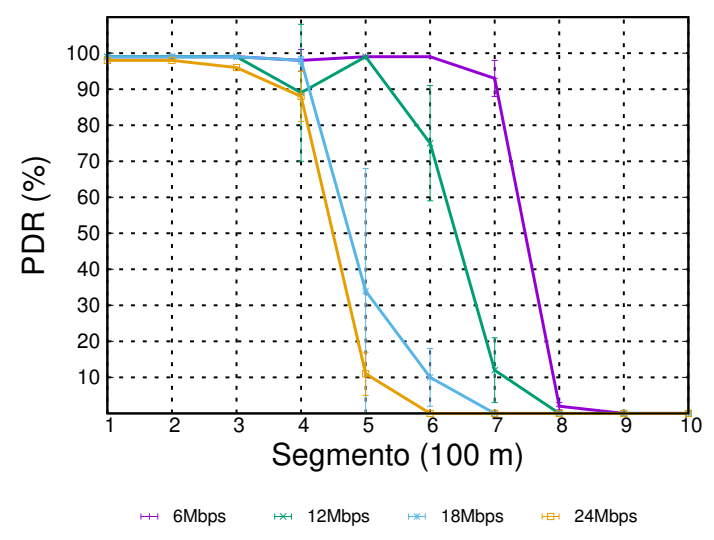

(b) PDR obtido dos experimentos práticos.

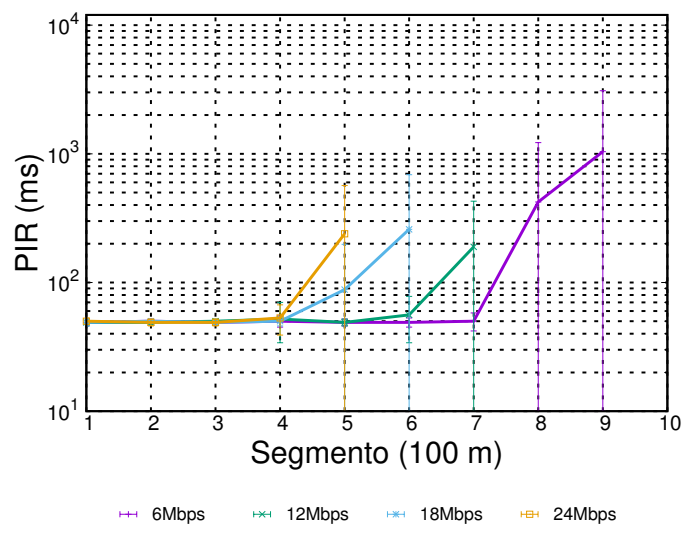

(d) PIR obtido dos experimentos práticos.

Figura 3. PDR e PIR obtidas no Cenário 1.

Como pode-se observar, o alcance máximo teórico de 1.000 m não é suportado por nenhuma modulação avaliada nos experimentos. Apenas a modulação associada à taxa de $6 \mathrm{Mbps}$ oferece um alcance máximo de aproximadamente $800 \mathrm{~m}$, em ambos os ambientes. Apesar disso, a PDR desta taxa demonstra que, embora seja possível receber pacotes a esta distância, o índice de 3.9\% nas simulações e 2.2\% nas medições reais seguramente não permitiria a uma aplicação funcionar satisfatoriamente. Em contrapartida, a uma distância de $700 \mathrm{~m}$, a PDR é consideravelmente maior, superior a 90\% em ambos os ambientes. Este comportamento de queda da PDR com o aumento da distância entre transmissor e receptor é similar ao obtido nas medições reais realizadas em [Sassi et al. 2017]. Naquele trabalho, à uma distância de $1.000 \mathrm{~m}$, calculou-se uma PDR de aproximadamente $25 \%$ à taxa de $6 \mathrm{Mbps}$, enquanto que à $700 \mathrm{~m}$ este valor passou para cerca de $94 \%$.

Analisando a influência da modulação correspondente à maior taxa de dados (24 Mbps), os resultados da PDR em ambos os ambientes sugerem que este esquema deve ser considerado apenas quando transmissor e receptor estejam geograficamente próximos 
um do outro. Mais perceptível nas simulações, já a partir de $200 \mathrm{~m}$ de afastamento, o índice da PDR passa de $99.9 \%$ para aproximadamente $0.2 \%$, enquanto que nos experimentos reais a PDR passa de $88.9 \%$ para $11.1 \%$ a partir de $400 \mathrm{~m}$. Novamente, os resultados práticos obtidos em [Sassi et al. 2017] apresentam um comportamento similar ao obtido no presente trabalho. Naquele trabalho, analisando a taxa de $24 \mathrm{Mbps}$, a PDR salta de $75 \%$ à 200 m para $25 \%$ à $400 \mathrm{~m}$, aproximadamente. No presente trabalho, este comportamento também pode ser observado nos dois ambientes para as taxas de 18 e $12 \mathrm{Mbps}$, sendo, entretanto, mais acentuado nas simulações. É possível concluir que, apesar de as curvas mostradas em ambos os gráficos apresentarem o mesmo comportamento, apenas os resultados relacionados à modulação associada à taxa de $6 \mathrm{Mbps}$ (valor de referência) são iguais em valor. Nas outras modulações, os valores obtidos para a PDR indicam que o alcance de comunicação é maior do que o indicado pelas simulações no NS-3.

Já com relação ao PIR obtido nas simulações, analisando as modulações associadas às taxas de $6 \mathrm{Mbps}$ e $12 \mathrm{Mbps}$ nos segmentos 8 e 5 , os valores obtidos destoam quando comparados com os valores dos demais segmentos. Entretanto, ao comparar as Figuras 3(c) e 3(a), percebe-se que estes resultados estão de acordo com a PDR obtido nos mesmos segmentos para as mesmas modulações. A explicação é que, nas modulações mais baixas, existe uma maior possibilidade de se obter uma comunicação a uma distância maior. Consequentemente, quanto maior for o alcance desta comunicação, pior será a qualidade da transmissão naquele ponto mais distante, prejudicando a PDR e, por consequência, o PIR. Este comportamento também pode ser percebido nos experimentos práticos. Entretanto, diferente das simulações, neste ambiente o PIR é afetado em todos os cenários (independente da modulação), indicando assim um compromisso mais forte entre a degradação da PDR e o aumento do PIR, tal como observado em [ElBatt et al. 2006].

De forma geral, os resultados deste primeiro conjunto de experimentos simulados e reais apontam uma notável degradação da PDR em ambos os ambientes à medida que a distância entre a OBU e a RSU aumenta. Dado que a potência de transmissão é fixa, à medida que se aumenta a distância entre transmissor e receptor, piora a qualidade do sinal recebido, independentemente da modulação adotada. Tal compromisso é prejudicial para modulações associadas a altas taxas de dados, visto que, quanto mais alta é a taxa, maiores são as chances de que ocorram falhas de integridade entre símbolos. Estes resultados podem significar um alerta para o funcionamento de aplicações cujo principal requisito é a comunicação a longas distâncias. Apesar de atender a este requisito, a incidência de blackouts de consciência situacional pode prejudicar a precisão da aplicação, oferecendo um risco à segurança dos participantes da VANET [Renda et al. 2016].

Apenas para esclarecer se o alcance máximo do IEEE 802.11p é equivalente ao valor teórico, o cenário anterior foi executado no mundo real com a taxa de $3 \mathrm{Mbps}$. Três rodadas em cada segmento foram executadas, e a Tabela 2 apresenta os resultados da análise. Como em [Sassi et al. 2017], é possível haver comunicação mesmo em distâncias superiores a $1.000 \mathrm{~m}$. Porém, devido à forte atenuação gerada pelo aumento da distância, a PDR passa a não ser satisfatória a partir do segmento 11 (46\%), alcançando apenas 3\% à uma distância de $1.200 \mathrm{~m}$, tornando inviável o funcionamento de qualquer aplicação.

\subsection{Efeito da Mobilidade Moderada na Comunicação}

Ao contrário do experimento anterior, neste conjunto de experimentos as métricas PDR e PIR são calculadas como médias gerais, e não com base nos resultados obtidos a 
Tabela 2. Alcance máximo real do padrão IEEE 802.11p.

\begin{tabular}{|c|c|c|c|c|c|c|c|c|c|c|c|c|c|}
\hline Segmento (ID) & 0 & 1 & 2 & 3 & 4 & 5 & 6 & 7 & 8 & 9 & 10 & 11 & 12 \\
\hline PDR (\%) & 100,0 & 99,8 & 99,53 & 99,8 & 99,8 & 99,5 & 99,8 & 99,8 & 99,8 & 92,2 & 66,4 & 46,0 & 0,03 \\
\hline
\end{tabular}

cada $100 \mathrm{~m}$. Assim, torna-se necessário desconsiderar transmissões realizadas em pontos da via onde, de acordo com as Figuras 3(a) e 3(b), notoriamente não existe comunicação com a RSU. Desta forma, definiu-se que o cálculo da PDR e do PIR será realizado com base nas transmissões iniciadas a partir do segmento da via onde a modulação associada à mais baixa taxa de dados considerada em nossos experimentos (6 Mbps) proporciona uma PDR superior a 70\%. Portanto, tanto nas simulações quanto nos experimentos práticos, o cálculo da PDR e PIR no cenário V2I será realizado apenas se as transmissões foram efetivadas a $700 \mathrm{~m}$ ou menos de distância.

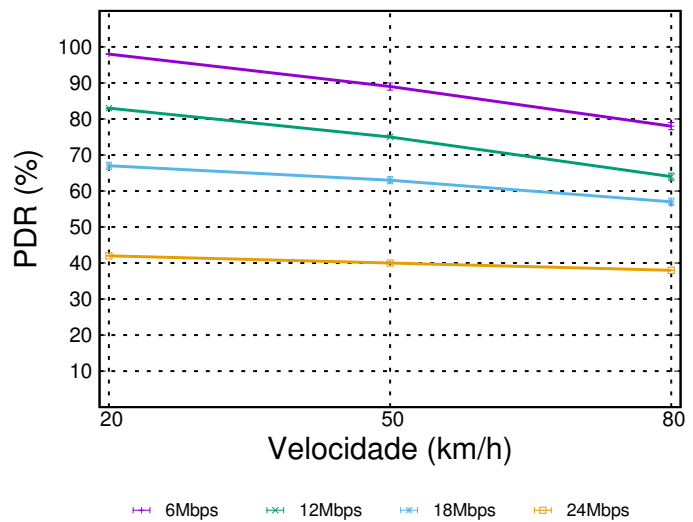

(a) PDR obtido das simulações.

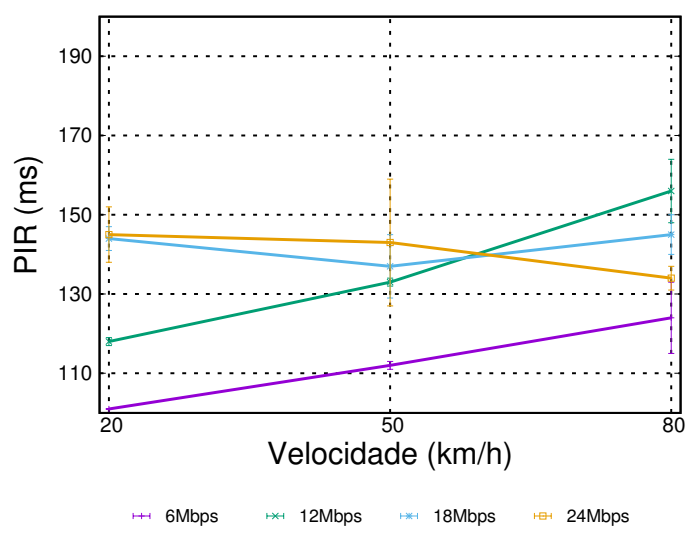

(c) PIR obtido das simulações.

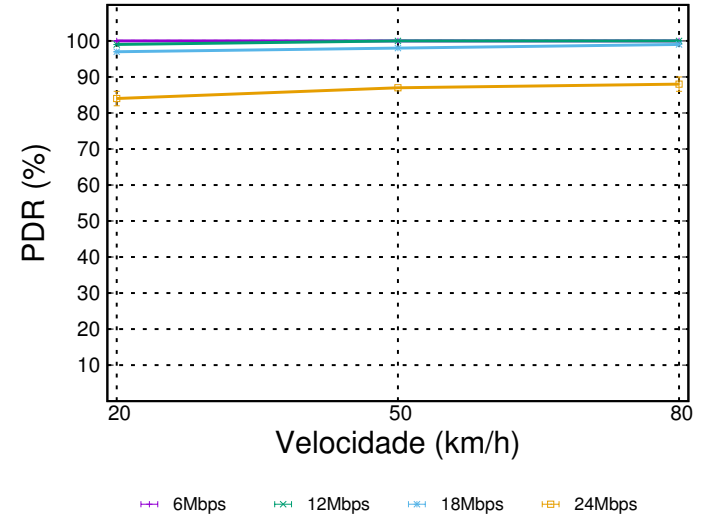

(b) PDR obtido dos experimentos práticos.

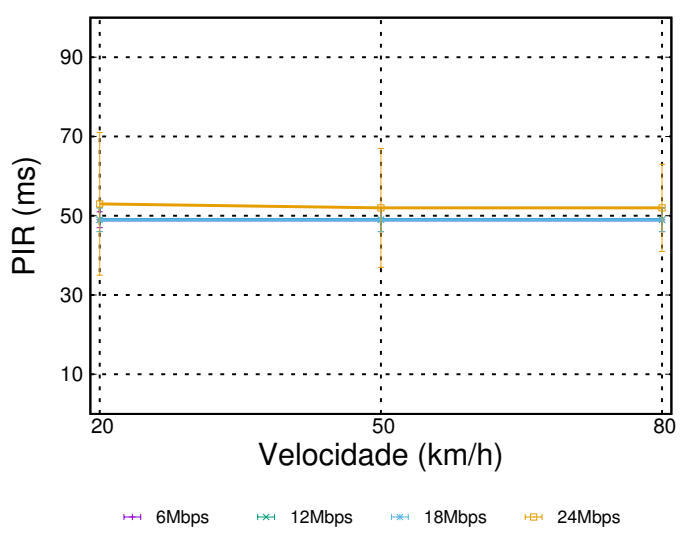

(d) PIR obtido dos experimentos práticos.

Figura 4. PDR e PIR obtidas no Cenário 2.

As Figuras 4(a) e 4(b) apresentam o efeito da mobilidade moderada na PDR da comunicação V2I nas simulações e nos experimentos reais. Como observado em [Sassi et al. 2017], nas Figuras 3(a) e 3(b) é possível perceber o efeito negativo provocado pelo aumento da taxa de dados na PDR em ambos os ambientes, apesar de este ser mais intenso nas simulações. Ainda nas simulações, pode-se observar também que, para qualquer modulação, ao aumentar a velocidade do veículo há uma degradação das métricas avaliadas. Este resultado pode ser atribuído ao impacto provocado pelo efeito Doppler 
nas ondas emitidas por objetos em movimento. Tal degradação é mais sutil quando analisados os resultados da taxa de $24 \mathrm{Mbps}$, tornando-se cada vez mais severa nas taxas mais baixas (18, 12 e 6 Mbps). Já nos experimentos reais, diferente do que ocorre em [Sassi et al. 2017], surpreendentemente esta degradação não é observada: os resultados deste ambiente demonstram que a PDR não é afetado pela velocidade do veículo. Como esperado (baseado nos resultados do cenário anterior) os resultados da PDR obtidos neste ambiente são substancialmente melhores do que os obtidos nas simulações. Não há, portanto, uma consistência entre os resultados das simulações e experimentos reais quando considerado o efeito da mobilidade moderada na PDR da comunicação V2I.

As Figuras 4(c) e 4(d) apresentam os resultados de PIR das simulações e medições reais. Nas simulações, é possível observar que, para as modulações associadas às taxas de 6 e $12 \mathrm{Mbps}$, o PIR é diretamente influenciado pela mobilidade: quanto maior a velocidade do veículo, maior é o tempo entre recepções de pacotes. Este comportamento, entretanto, não foi observado nas modulações associadas às taxas de 18 e $24 \mathrm{Mbps}$. Para estas modulações, mesmo que a PDR seja maior nas velocidades mais baixas, o PIR, inesperadamente, também pode ser maior. Como a $20 \mathrm{~km} / \mathrm{h}$ é possível alcançar um maior alcance na comunicação devido ao menor impacto do efeito Doppler, usando estas modulações, os pacotes transmitidos podem ser recebidos em tempos mais espaçados devido aos problemas de integridade associados com transmissões às taxas mais altas a maiores distâncias. Desta maneira, o PIR resultante deste cenário será maior mesmo na velocidade de 20 $\mathrm{km} / \mathrm{h}$. Ao mesmo tempo, ao aumentar a velocidade do veículo (para $50 \mathrm{~km} / \mathrm{h}$ e $80 \mathrm{~km} / \mathrm{h}$ ), pode acontecer de, mesmo que a PDR seja muito baixa (até mesmo menor que no cenário com velocidade de $20 \mathrm{~km} / \mathrm{h}$ ), os poucos pacotes recebidos pelo receptor serem recebidos em lote, de maneira quase contínua (como se transmitidos quando o transmissor se encontrava próximo do receptor). Tal cenário implicaria em um PIR quase proporcional à taxa de transmissão de pacotes, mesmo que a PDR seja baixa. Esta hipótese foi vislumbrada em [Renda et al. 2016]. Para as taxas de 6 e $12 \mathrm{Mbps}$, este comportamento não é refletido. Neste caso, a tendência é que a maioria dos pacotes enviados sejam recebidos com sucesso, mesmo quando há um alcance maior na comunicação.

Já nos experimentos práticos, pode-se considerar que o PIR não é influenciado nem pelas diferentes taxas de dados, nem pelo aumento da velocidade do veículo, visto que os tempos de PIR obtidos em cada modulação se encontram em um intervalo muito próximo, de aproximadamente $50 \mathrm{~ms}$. Apesar disso, os resultados obtidos nos experimentos práticos são mais coerentes que os obtidos nas simulações. Nesse ambiente, os resultados apontam um ligeiro compromisso entre o PIR e a PDR na comunicação V2I, dado que, à medida que a PDR aumenta, ligeiramente o PIR diminui. Este comportamento independe da modulação avaliada, porém é mais claramente percebido a 24 Mbps.

Comparando os ambientes, não foi possível obter uma equivalência total com relação aos resultados de PIR. Nas simulações, apenas as modulações associadas às taxas de 6 e $12 \mathrm{Mbps}$ apresentam resultados condizentes com o esperado. Os resultados também demonstram que, como identificado em [Renda et al. 2016], pode não ser possível estimar confiavelmente os valores de PIR com base nos resultados da PDR. Além disso, a ausência de um padrão de entrega dos pacotes transmitidos sob efeito dos modelos de perda de propagação no NS-3 sugere a necessidade de revisão dos referidos modelos. 


\subsection{Efeito da Mobilidade Intensa na Comunicação}

Neste último conjunto de experimentos, dois veículos viajando em direções opostas variam sua velocidade entre $20 \mathrm{~km} / \mathrm{h}, 50 \mathrm{~km} / \mathrm{h}$ e $80 \mathrm{~km} / \mathrm{h}$, obtendo velocidades relativas de $40 \mathrm{~km} / \mathrm{h}, 100 \mathrm{~km} / \mathrm{h}$ e $160 \mathrm{~km} / \mathrm{h}$. Novamente, calcula-se PDR e PIR como médias gerais.

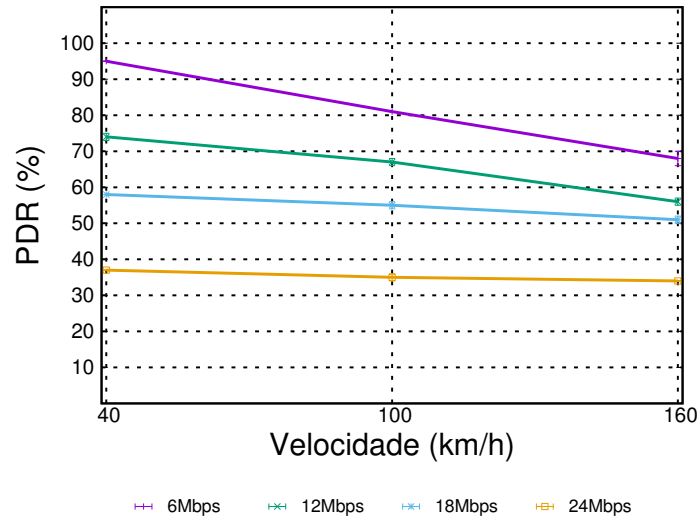

(a) PDR obtido das simulações.

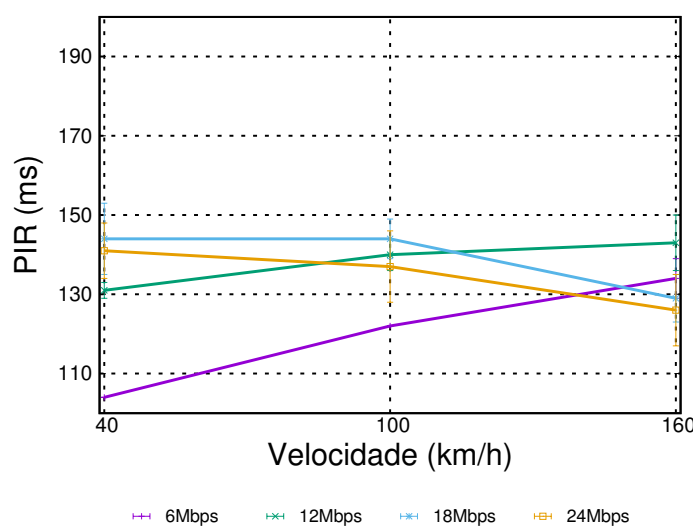

(c) PIR obtido das simulações.

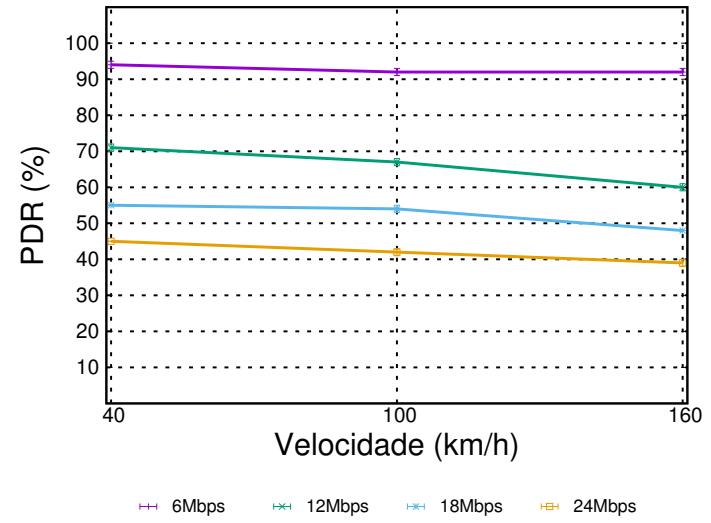

(b) PDR obtido dos experimentos práticos.

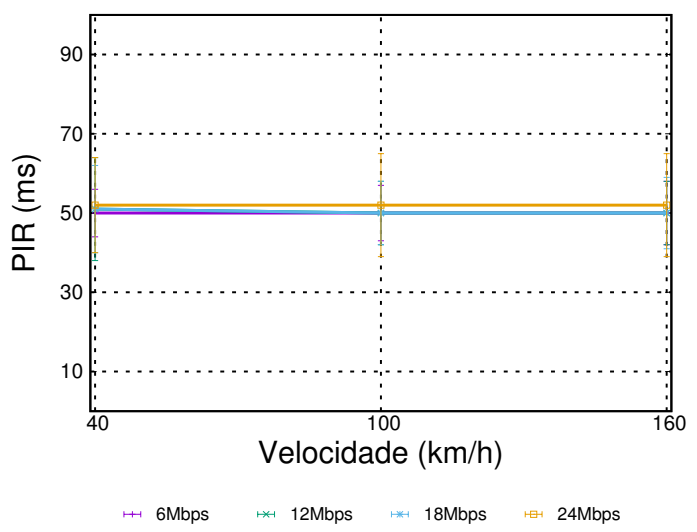

(d) PDR obtido dos experimentos práticos.

Figura 5. PDR e PIR obtidas no Cenário 3.

Os resultados relacionados ao efeito da mobilidade intensa na PDR das simulações e resultados práticos são apresentados nas Figuras 5(a) e 5(b). Comparado ao cenário anterior, a velocidade relativa provocada pela alta mobilidade de ambos os veículos definitivamente proporciona uma piora na PDR de ambos os ambientes, independente da modulação. Nas simulações, em alguns casos esta diferença chega a 10\% (modulação associada à taxa de $6 \mathrm{Mbps}$ e $160 \mathrm{~km} / \mathrm{h}$ ), enquanto que nos experimentos práticos a diferença alcança até $51 \%$ (modulação associada à taxa de $18 \mathrm{Mbps}$ e $160 \mathrm{~km} / \mathrm{h}$ ). Apesar de algumas diferenças, como a degradação da PDR é mais intensa a $6 \mathrm{Mbps}$ nas simulações, enquanto nos experimentos práticos ocorre o inverso, percebe-se que as curvas mostradas nos gráficos de ambos os ambientes são similares, apresentando o mesmo comportamento. Desta vez, estes resultados concordam com aqueles obtidos em [Sassi et al. 2017], mesmo que, no presente trabalho, as curvas representando as mais altas taxas (12, 18 e $24 \mathrm{Mbps})$ não apresentem o mesmo afunilamento. Em [Sassi et al. 2017], nas transmissões realizadas à $24 \mathrm{Mbps}$ e com o veículo trafegando a $40 \mathrm{~km} / \mathrm{h}$ e $160 \mathrm{~km} / \mathrm{h}$, é possível alcançar, respectivamente, uma PDR de aproximadamente $92 \%$ e $75 \%$. Já no presente trabalho, as 
transmissões realizadas à esta mesma taxa alcançam uma PDR de cerca de $45 \%$ e $39 \%$, respectivamente.

Já as Figuras 5(c) e 5(d) apresentam o PIR obtido nas simulações e experimentos práticos. Como mostrou a Figura 4(c), nas simulações o PIR foi diretamente influenciado pela mobilidade nas modulações associadas às taxas de 6 e $12 \mathrm{Mbps}$. Novamente, para estas taxas, quanto maior a velocidade do veículo, maior é o tempo entre recepções de pacotes. Entretanto, baseado na mesma explicação descrita no cenário anterior, nas taxas de 18 e $24 \mathrm{Mbps}$, o PIR é diretamente influenciado pela maior probabilidade de erros devido a transmissões realizadas a longa distâncias. Já nos experimentos práticos, como mostrou a Figura 4(d), pode-se considerar que o PIR não é influenciado nem pelo aumento da taxa de dados, nem pelo aumento da velocidade do veículo. Entretanto, neste cenário, não é possível perceber sequer um ligeiro compromisso entre o PIR e a PDR na comunicação. Pelo contrário, mesmo que os tempos de PIR para cada modulação estejam em um intervalo muito próximo, percebe-se que, para algumas modulações avaliadas detalhadamente (como $18 \mathrm{Mbps}$ ), à medida que a PDR diminui, o PIR também diminui. Como no cenário V2I, não há equivalência entre os resultados de PIR obtidos nas simulações e experimentos práticos considerando a comunicação $\mathrm{V} 2 \mathrm{~V}$.

\section{Conclusões e Trabalhos Futuros}

Este trabalho apresentou uma comparação entre os resultados da avaliação do padrão IEEE 802.11 p obtidos por meio de simulações realizadas no NS-3 e medições reais utilizando OBUs e RSUs comerciais. O objetivo era preencher uma lacuna da literatura, investigando as possíveis correspondências entre dados coletados em ambos os ambientes. Em cenários envolvendo comunicações V2I e V2V, o impacto de diferentes modulações e velocidades foi analisado, e os resultados do alcance máximo da comunicação, da taxa de entrega de pacotes e do tempo entre recepções de pacotes foram confrontados.

Os resultados revelam que apenas a modulação associada à taxa de $6 \mathrm{Mbps}$ oferece um alcance satisfatório de $700 \mathrm{~m}$, o que sugere o uso de baixas taxas ao visar comunicações de longo alcance. Apenas este resultado é igual nos dois ambientes. Nas outras modulações, o alcance foi maior no mundo real. É nítido o impacto do aumento da distância e da taxa de dados na PDR. Quanto ao impacto da mobilidade moderada na comunicação, não há uma consistência entre os resultados. Enquanto nas simulações o aumento da velocidade causa uma degradação da PDR, nas medições reais isto não foi observado. Já a mobilidade intensa afetou a comunicação de ambos os ambientes.

Com relação ao PIR, os valores obtidos na avaliação do alcance indicam um compromisso com a PDR, mais notório nas medições reais. Quanto ao impacto da mobilidade moderada na comunicação, não houve uma equivalência total. Nas simulações, apenas as taxas de 6 e $12 \mathrm{Mbps}$ apresentam resultados condizentes, com o PIR sendo influenciado pela mobilidade. Já nas medições reais, considera-se que o PIR não é influenciado pelas diferentes taxas ou aumento da velocidade. Estes resultados também são obtidos em ambos os ambientes ao analisar o impacto da mobilidade intensa na comunicação, reforçando a impossibilidade de estimar confiavelmente o PIR com base na PDR.

As diferenças encontradas nos resultados dos dois ambientes indicam a necessidade de aprimorar os modelos usados em simulações de redes veiculares. Como trabalhos futuros, pretende-se investigar o impacto de uma rede veicular mais densa, além das dife- 
rentes condições de LoS e NLoS (Non-Line-of-Sight) no desempenho do IEEE 802.11p. Pretende-se, ainda, comparar os resultados destas medições reais com aqueles obtidos por meio de simulações realizadas no simulador Veins (Vehicles in Network Simulation).

\section{Agradecimentos}

Este trabalho foi parcialmente financiado pela CAPES, CNPq, FAPERJ e pelos processos n ${ }^{0}$ 15/24494-8 e no 15/24490-2, da Fundação de Amparo à Pesquisa do Estado de São Paulo (FAPESP).

\section{Referências}

Amarante, T. C., Barcelos, V. P., D’Alessandre, L. D., and Correia, L. H. A. (2015). Deteção Automática e Alerta de Acidentes em Redes Veiculares Reais. In SBRC'2015, pages 146-159, Vitória, ES.

Arbabi, H. and Weigle, M. C. (2010). Highway Mobility and Vehicular Ad-Hoc Networks in ns-3. In Proceedings of the Winter Simulation Conference.

Barcelos, V. P., Amarante, T. C., Drury, C. D., and Correia, L. H. A. (2014). Sistema de monitoramento de veículos usando dispositivos no padrao IEEE 802.11p. In SBRC'2014, pages 939-951, Florianópolis, Santa Catarina.

DATASUS (2017). Indicadores de Efetividade da Política Nacional de Mobilidade Urbana. http://www.cidades.gov.br/component/content/article?id=4965. Acessado em dezembro de 2017.

ElBatt, T., Goel, S. K., Holland, G., Krishnan, H., and Parikh, J. (2006). Cooperative Collision Warning Using Dedicated Short Range Wireless Communications. In Proceedings of the 3rd International Workshop on Vehicular Ad Hoc Networks, VANET '06, pages 1-9, New York, NY, USA. ACM.

Gräfling, S., Mähönen, P., and Riihijärvi, J. (2010). Performance evaluation of IEEE 1609 WAVE and IEEE $802.11 \mathrm{p}$ for vehicular communications. In Ubiquitous and Future Networks (ICUFN), 2010 Second International Conference on, pages 344-348. IEEE.

Jafari, A., Al-Khayatt, S., and Dogman, A. (2012). Performance evaluation of IEEE 802.11 p for vehicular communication networks. In Communication Systems, Networks \& Digital Signal Processing (CSNDSP), 2012 8th International Symposium on, pages 1-5. IEEE.

OMS (2017). Road injuries. http://who.int/mediacentre/factsheets/fs358. Acessado em dezembro de 2017.

Pinto, J. B., Gomes, L. C., Castanho, E. M., Campista, M. E. M., and Costa, L. H. M. K. (2016). Um Algoritmo para Cálculo de Distância Segura de Frenagem para Prevenção de Colisão Dianteira em Redes Veiculares. In SBRC'2016, pages 59-72, Salvador, Bahia.

Renda, M. E., Resta, G., Santi, P., Martelli, F., and Franchini, A. (2016). IEEE 802.11p VANets: Experimental evaluation of packet inter-reception time. Computer Communications, 75:26 - 38.

Sabouni, R. and Hafez, R. M. (2012). Performance of DSRC for V2V communications in urban and highway environments. In Electrical \& Computer Engineering (CCECE), 2012 25th IEEE Canadian Conference on, pages 1-5. IEEE.

Sassi, A., Elhillali, Y., and Charfi, F. (2017). Evaluating Experimental Measurements of the IEEE 802.11 p Communication Using ARADA LocoMate OBU Device Compared to the Theoretical Simulation Results. Wireless Personal Communications, 97(3):3861-3874.

Schrank, D., Eisele, B., Lomax, T., and Bak, J. (2015). 2015 urban mobility scorecard.

Teixeira, F. A., e Silva, V. F., Leoni, J. L., Macedo, D. F., and Nogueira, J. M. (2014). Vehicular networks using the IEEE 802.11p standard: An experimental analysis. Vehicular Communications, 1(2):91 - 96.

Vivek, N., Srikanth, S., Saurabh, P., Vamsi, T., and Raju, K. (2014). On field performance analysis of IEEE $802.11 \mathrm{p}$ and WAVE protocol stack for V2V \& V2I communication. In Information Communication and Embedded Systems (ICICES), 2014 International Conference on, pages 1-6. IEEE.

Wang, Y., Hu, J., Zhang, Y., and Xu, C. (2015). Reliability evaluation of IEEE 802.11p-based vehicle-tovehicle communication in an urban expressway. Tsinghua Science and Technology, 20(4):417-428.

Zhao, Z., Cheng, X., Wen, M., Jiao, B., and Wang, C.-X. (2013). Channel estimation schemes for IEEE $802.11 \mathrm{p}$ standard. IEEE Intelligent Transportation Systems Magazine, 5(4):38-49. 